\title{
The influence of the ammonia concentration and the water content on the water sorption behavior of ambient pressure dried silica xerogels
}

\author{
${\text { Lukas Huber } \mathbb{D}^{1} \cdot \text { Silvia Paz Comesaña }}^{1} \cdot$ Matthias M. Koebel ${ }^{1}$
}

Published online: 29 July 2020

(c) The Author(s) 2020

\section{Abstract}

Porous silica xerogels were synthesized within $10 \mathrm{~h}$ by a two-step sol-gel process under atmospheric conditions. In the first step, tetraethylorthosiloxane (TEOS) was hydrolyzed with water using sulfuric acid as a catalyst. In the second step, water and ammonia were added to the prehydrolyzed silica sol prior to the drying of the samples at $150{ }^{\circ} \mathrm{C}$. The influence of the ammonia concentration and the water content on the physicochemical properties and the water sorption behavior of silica xerogels produced in the aforementioned way was investigated. The resulting silica xerogels were characterized by helium pycnometry, scanning electron microscopy, fourier-transform infrared spectroscopy, nitrogen sorption, and water sorption. It was shown that a higher ammonia concentration leads to an increased pore size of the silica xerogel which starts to adsorb water at a higher relative pressure. With an increased water content during gelation, the silica xerogel has a higher specific surface area and a higher water sorption capacity. Furthermore, silica xerogels can be tailored by a controlled addition of ammonia and water to have a higher water sorption capacity than the reference silica gel over the whole range of relative pressure.

\section{Graphical Abstract}
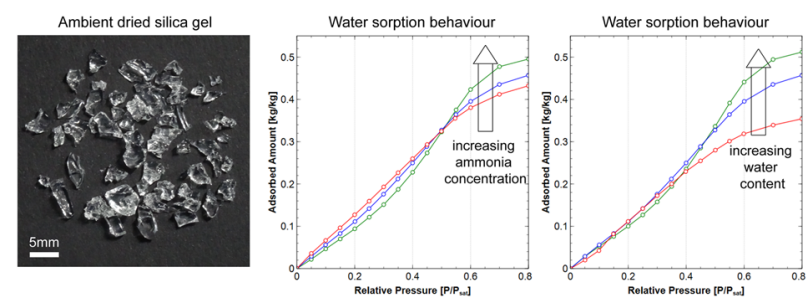

Keywords Adsorption $\cdot$ Silica gel $\cdot$ Xerogel $\cdot$ Water sorption $\cdot$ Sorben

Supplementary Information Supplementary information The online version of this article (https://doi.org/10.1007/s10971-02005349-1) contains supplementary material, which is available to authorized users.

\footnotetext{
Matthias M. Koebel

Matthias.Koebel@empa.ch

1 Empa, Swiss Federal Laboratories for Materials Science and Technology, Laboratory for Building Energy Materials and Components, Überlandstrasse 129, CH-8600

Dübendorf, Switzerland
} 


\section{Highlights}

- Silica xerogels were synthesized within $10 \mathrm{~h}$ by a hydrolysis-condensation sol-gel process.

- An increased ammonia concentration during the condensation reaction leads to a higher porosity and larger average pore size of the silica xerogel.

- A higher water content during the condensation reaction increases the specific surface area and the water sorption capacity of the silica xerogel.

\section{Introduction}

Adsorption heat pumps (AHPs) have gained a lot of interest in the past couple of decades since they are considered an environmentally friendly and cost-effective alternative to compressor-based heat pumps. AHPs utilize waste heat rather than electrical energy to provide space heating and cooling. Conventional AHPs are equipped with silica gel as an adsorbent, however, the widespread application of AHPs has not been achieved due to the low water adsorptivity and the poor mass and heat transport properties of silica gels [1]. The synthesis and structural characterization of porous solids, such as zeolites, metal-organic frameworks, activated carbons, or silica gels, have received a growing interest and an increase in research studies [2-7]. In order to maximize efficiency, the amount of cycled water within the operation window has to be maximized meaning that the sorbent has to be tailored for its application. To address this issue, silica gels with tailored porosity and pore size have been proposed to enhance mass transport properties.

An attractive method to tailor the pore size of the silica xerogel is by varying the concentration of acid or base used for hydrolysis or condensation respectively. It was found that xerogels synthesized with a low concentration of acid and base have smaller particle and pore size than those synthesized with a high concentration of acid and base $[8,9]$. Alcañiz et al. [2] investigated the influence of the pore size distribution of silica xerogels on the water sorption behavior. It was found that essentially microporous silica gels adsorb water at low and medium relative pressures $(0<$ $\left.\mathrm{P} / \mathrm{P}_{\mathrm{sat}}<0.6\right)$ whereas silica gels which contain micro- and mesoporosity adsorb water in the whole pressure range $(0<$ $\left.\mathrm{P} / \mathrm{P}_{\text {sat }}<0.95\right)$.

The mechanism of adsorption of water on silica gels has been investigated intensively within the last decades [1016]. This mechanism is rather complex due to the polarity of the water molecule. It is known that the surface of amorphous, hydroxylated silica is covered with silanol ( $\equiv \mathrm{Si}-\mathrm{OH})$ groups. When the dried silica surface is exposed to water vapor, the water molecules start to adsorb, presumably by direct interaction with the silanols [17]. However, the sequence of the adsorption of water on the silica surface is not yet clear. One option for the water molecule to adsorb is "oxygen down" and by the formation of a 1:1 complex in which the surface $\mathrm{OH}$ groups are donors and the water molecules are acceptors of the hydrogen bond [18]. Another option is the water interacts with $\mathrm{SiOH}$ as a hydrogen acceptor which means that the water molecule is doubly hydrogen bonded to the surface [19-21]. At high relative humidity, the water molecules condense at the entrance to the pores before the condensed liquid will migrate into the pores, a process known as capillary condensation $[13,22,23]$.

Water vapor adsorption on silica gel has been investigated intensively owing to the fact that silica gel is used in industry as a drying agent, catalyst support, or filler $[11,24-26]$. Those studies focus on the cycle stability towards water, diffusion times, or the kinetics of water adsorption on silica gel. Other studies focus on the synthesis of the silica gels and the subsequent characterization by density measurements, fourier-transform infrared spectroscopy, and nitrogen sorption analysis $[8,9]$. There exists only a few studies that investigate the influence of the pore size on the water sorption behavior through inhouse synthesis of silica gels [2, 27].

In this work, we report the synthesis of silica xerogels derived from tetraethylorthosiloxane (TEOS) within $10 \mathrm{~h}$ including hydrolysis, gelation, aging and drying. The effect of the ammonia concentration and the water content on the porosity, pore size, surface area, and the water sorption behavior were examined. It was found that a higher concentration of ammonia during the condensation reaction leads to a bigger pore size and shifts the water sorption isotherm to high relative pressure. The water sorption capacity can be increased by increasing the amount of water during the condensation reaction. By a controlled addition of ammonia and water, silica xerogels can be tailored to have a higher water sorption capacity than the reference silica gel over the whole range of relative pressure.

\section{Experimental}

\subsection{Materials}

Ethanol (A15-A ethanol denatured with 2\% methyl ethyl ketone) was obtained from Alcosuisse. Ammonium hydroxide (29 wt.\%) and sulfuric acid (95-98 wt.\%) were received from Sigma Aldrich. Tetraethylorthosiloxane (TEOS, $\geq 98 \%$ ) was received from Evonik. All solutions 
Table 1 Table of experiments

\begin{tabular}{llll}
\hline Sample & Prehydrolyzed silica sol [ml] & $\mathrm{H}_{2} \mathrm{O}[\mathrm{ml}]$ & Ammonia [mM] \\
\hline XGW1 & 41 & 4.04 & 18.3 \\
XGW2 & 41 & 4.04 & 36.6 \\
XGW3* & 41 & 4.04 & 61.1 \\
XGW4 & 41 & 4.04 & 91.6 \\
XGW5 & 41 & 4.04 & 122.1 \\
XGA6 & 41 & 2.04 & 61.1 \\
XGA7 & 41 & 3.04 & 61.1 \\
XGA8* & 41 & 4.04 & 61.1 \\
XGA9 & 41 & 5.04 & 61.1 \\
XGA10 & 41 & 6.04 & 61.1
\end{tabular}

XGW: silica xerogels series synthesized with the same content of water

XGA: silica xerogels series synthesized with the same concentration of ammonia

${ }^{*} \mathrm{XGW} 3$ and XGA8 are the same sample

were prepared from ultrapure water (double distilled quality, $>18 \mathrm{M} \Omega \cdot \mathrm{cm}$ ). As reference adsorbents, commercial silica gel beads (RD-type) with a diameter between 1.8 and $2.0 \mathrm{~mm}$ were received from Fuji Silysia Chemical Ltd.

\subsection{Preparation of the xerogels}

The synthesis of the silica xerogels consists out of three steps: hydrolysis, condensation, and drying. For the hydrolysis, $18 \mathrm{~g}$ of ethanol were mixed with $0.256 \mathrm{~g}$ of concentrated $\mathrm{H}_{2} \mathrm{SO}_{4}$. This solution was added to $308 \mathrm{~g}$ of TEOS and stirred vigorously for $1 \mathrm{~h}$ at room temperature. Then, the temperature was increased to $35^{\circ} \mathrm{C}$ before $41.62 \mathrm{~g}$ of water was added over the course of $2 \mathrm{~h}$ using a syringe pump. The obtained prehydrolyzed silica sol was then used for the condensation reaction.

For condensation, $41 \mathrm{ml}$ of the prehydrolyzed silica sol was mixed with water before the addition of ammonia (Table 1). The silica sol was poured into plastic containers and sealed before being put in an oven at $65^{\circ} \mathrm{C}$. The gelation onset was after about 5-10 min from the addition of the last reagent. After the gelation, the gels were covered with $10 \mathrm{ml}$ of Ethanol and placed back in the oven for $6 \mathrm{~h}$ of aging. Then, the gels were removed from the plastic mold and dried in a ventilation oven at $150{ }^{\circ} \mathrm{C}$ for $2 \mathrm{~h}$. Before the characterization, the dried xerogels were placed in an $80 \%$ relative humidity chamber for 10 days in order to exchange the ethoxy groups by hydroxyl groups.

\subsection{Characterization}

The envelope density ( $\rho$ ) was measured by a Geopyc 1360 from Micromeritics. In this characterization method, the silica xerogel (2-4 mm in diameter) was placed in a bed of DryFlo $^{\circledR}$ granular medium, which was agitated and carefully consolidated around the scaffold using a piston [28]. The sample was getting consolidated by agitation and rotation of the cylindrical chamber since the piston was gradually pushed into the chamber until the consolidation force was reached, followed by retraction and recompression. A $12.7 \mathrm{~mm}$ diameter chamber was used to measure with a conversion factor of 0.1246 and a consolidation force of $4 \mathrm{~N}$. For each measurement, 10 cycles were carried out. The skeleton density $\left(\rho_{\text {skeleton }}\right)$ was determined with an AccuPyc II 1340 helium pycnometer (Micromeritics, helium purity of 99.999\%). The skeleton volume of the sample was measured by detecting the change in pressure owing to the volume of helium that was displaced by the silica xerogel within the sealed and pressure-equilibrated chamber. Each measurement consisted of 10 purge cycles and 10 analysis cycles. Prior to all density measurements, the silica xerogels were dried for $12 \mathrm{~h}$ at $150^{\circ} \mathrm{C}$. The porosity $\Phi$ was calculated from the envelope density $\rho$ and the skeleton density $\rho_{\text {skeleton }}$ using Eq. (1):

$\Phi=1-\frac{\rho}{\rho_{\text {skeleton }}}$

The pore volume $\left(\mathrm{V}_{\text {pore }}\right)$ was determined from Eq. (2):

$V_{\text {pore }}=\frac{1}{\rho}-\frac{1}{\rho_{\text {skeleton }}}$

Scanning electron microscopy (SEM) was performed for microstructural investigation after coating with $15-20 \mathrm{~nm}$ of Platinum. SEM images have been recorded with a FEI Nova NanoSEM 230 at an acceleration voltage of $7 \mathrm{kV}$ and a working distance of $5 \mathrm{~mm}$.

$\mathrm{N}_{2}$ adsorption and desorption isotherms at $77 \mathrm{~K}$ were collected on a Micromeritics 3flex Surface Area and Porosity Analyzer. Prior to the experiments, approximately $150 \mathrm{mg}$ of small silica pieces were degassed at $150{ }^{\circ} \mathrm{C}$ for $40 \mathrm{~h}$ at a pressure of $1.6 \times 10^{-2} \mathrm{mbar}$. The total surface area of the materials was calculated by the Brunauer-Emmet-Teller (BET) method [29]. A Physi ViewCalc tool from Micromeritics was used to calculate the micropore volume $\left(\mathrm{V}_{\text {Micro }}\right)$ with the t-plot method [30]. The total pore volume $\left(\mathrm{V}_{\text {total }}\right)$ was calculated from the volume of nitrogen adsorbed $\left(\mathrm{V}_{\mathrm{ads}}\right)$ converted to liquid $\mathrm{V}_{\text {liq }}$ at a pressure close to $\mathrm{P} / \mathrm{P}_{0}=1$ [31]. The mesopore volume $\left(\mathrm{V}_{\text {Meso }}\right)$ was calculated by subtracting the value of $\mathrm{V}_{\text {Micro }}$ from $\mathrm{V}_{\text {total }}$ [32]. The average pore diameter $\left(\mathrm{D}_{\text {pore }}\right)$ was calculated from the total pore volume $\left(\mathrm{V}_{\text {total }}\right)$ of the xerogel and its specific surface area $\left(\mathrm{S}_{\mathrm{BET}}\right)$ by $\mathrm{D}_{\text {pore }}=$ $4 \mathrm{~V}_{\text {pore }} / \mathrm{S}_{\mathrm{BET}}$. The pore size distributions were determined by the Barrett-Joyner-Halenda (BJH) method based on the desorption branch [33]. 
Fourier-transform infrared spectroscopy (FTIR) was performed on a Bruker Tensor 27 spectrometer in attenuated total reflectance (ATR) mode with a diamond analyzer crystal. Each spectrum was collected in the range from 4000 to $600 \mathrm{~cm}^{-1}$ with 32 scans. The spectra were obtained using the OPUS software provided by the instrument manufacturer (Bruker Optics $\mathrm{GmbH}$, Ettlingen, Germany). Prior to the measurements, the samples were degassed in a vacuum oven $(10 \mathrm{mbar})$ at $50{ }^{\circ} \mathrm{C}$ for $16 \mathrm{~h}$.

Dynamic vapor sorption (DVS) isotherms were measured by an isothermal gravimetric DVS apparatus (TA Instruments VTI-SA + ). The amount of adsorbed water vapor was continuously recorded by a high-precision microbalance with an accuracy of $10 \mu \mathrm{g}$. The amount of sample material placed in the experiment chamber was $10-30 \mathrm{mg}$. Prior to the actual measurement, the sample was heated to $90{ }^{\circ} \mathrm{C}$ under nitrogen atmosphere for $1 \mathrm{~h}$ and the resulting mass was taken as the dry reference mass. For the subsequent measurement, the sample was cooled down to $50^{\circ} \mathrm{C}$. Adsorption/desorption cycles were performed at a constant temperature of $50{ }^{\circ} \mathrm{C}$. The material is exposed to relative pressure $\left(\mathrm{P} / \mathrm{P}_{\text {sat }}\right)$ steps between 0 and 0.6 in 0.05 intervals and between 0.6 and 0.8 in 0.1 intervals in a controlled flow of a mixture of nitrogen and water vapor while the sample mass is recorded every $60 \mathrm{~s}$. At $50{ }^{\circ} \mathrm{C}$, the saturation pressure of water is $124 \mathrm{mbar}\left(\mathrm{P} / \mathrm{P}_{\text {sat }}=1\right)$. For each step, the pressure was kept constant until the rate of mass change became negligible (less than $0.08 \% / 5 \mathrm{~min}$ ) and the corresponding pressure and mass values were recorded until the entire adsorption and desorption isotherms were completed.

\section{Results and discussion}

\subsection{Density and porosity}

The influence of the catalyst concentration and the content of water on the envelope density, skeleton density, porosity, and calculated pore volume is shown in Table 2. It can be observed that a higher concentration of ammonia leads to a lower density and an increase in porosity as well as pore volume. These results are in agreement with the observations from Fidalgo et al. [8]. An increase in the ammonia concentration during gelation leads to a decrease in envelope density and an increase in porosity. Chan et al. [31] found that the pore volume is increasing when the gel is synthesized with a higher concentration of $\mathrm{NaF}$ which is in agreement with our observed data.

A decrease in the density is usually accompanied by a lower volume shrinkage during the drying of the material. The shrinkage is influenced by the responses of the structures to the removal of the solvent upon drying. Gels with a large bulk modulus are more likely to withstand the capillary pressure which leads to a lower density of the dried xerogel [8]. Possibly, gels which where synthesized with a high concentration of ammonia have a stronger network which leads to a lower shrinkage and, therefore, to a lower density of the dried xerogel.

Furthermore, an increase of the water content for the condensation reaction also leads to a reduction of the envelope density. According to Milea et al. [34], an increase in water content leads to a corresponding increase in condensation rate. A higher degree of condensation leads to a stronger silica network and, therefore, to a lower shrinkage during drying [35].

\subsection{Xerogel microstructure}

Figure 1 shows the SEM micrographs of the xerogels after drying at $150{ }^{\circ} \mathrm{C}$. It can be observed that the xerogels reveal a 3-dimensional network of primary particles [36]. A higher concentration of ammonia leads to a bigger particle size. This observation is consistent with the measurement of the envelope density. Increasing the ammonia concentration induces the formation of larger and more cross-linked polymers with a stronger network and a larger particle size.
Table 2 Effect of catalyst and water concentration on envelope density, skeleton density, porosity and, pore volume

\begin{tabular}{lllll}
\hline Sample & $\rho\left[\mathrm{g} / \mathrm{cm}^{3}\right]$ & $\rho_{\text {skeleton }}\left[\mathrm{g} / \mathrm{cm}^{3}\right]$ & Porosity $\Phi[\%]$ & $\mathrm{V}_{\text {pore }}\left[\mathrm{cm}^{3} / \mathrm{g}\right]$ \\
\hline XGW1 & $1.149 \pm 0.017$ & $1.978 \pm 0.001$ & 41.9 & $0.365 \pm 0.013$ \\
XGW2 & $1.065 \pm 0.011$ & $1.946 \pm 0.002$ & 45.3 & $0.425 \pm 0.010$ \\
XGW3 & $1.001 \pm 0.008$ & $1.973 \pm 0.002$ & 49.3 & $0.492 \pm 0.008$ \\
XGW4 & $0.985 \pm 0.006$ & $1.945 \pm 0.001$ & 49.3 & $0.501 \pm 0.006$ \\
XGW5 & $0.925 \pm 0.005$ & $1.944 \pm 0.001$ & 52.4 & $0.567 \pm 0.006$ \\
XGA6 & $1.083 \pm 0.007$ & $1.925 \pm 0.001$ & 43.8 & $0.404 \pm 0.006$ \\
XGA7 & $1.078 \pm 0.007$ & $1.927 \pm 0.001$ & 44.1 & $0.409 \pm 0.006$ \\
XGA8 & $1.001 \pm 0.008$ & $1.973 \pm 0.002$ & 49.3 & $0.492 \pm 0.008$ \\
XGA9 & $0.935 \pm 0.008$ & $1.962 \pm 0.001$ & 52.3 & $0.560 \pm 0.009$ \\
XGA10 & $0.940 \pm 0.004$ & $1.967 \pm 0.001$ & 52.2 & $0.555 \pm 0.005$ \\
Reference silica gel & $1.249 \pm 0.019$ & $2.060 \pm 0.002$ & 38.9 & $0.315 \pm 0.012$ \\
\hline
\end{tabular}



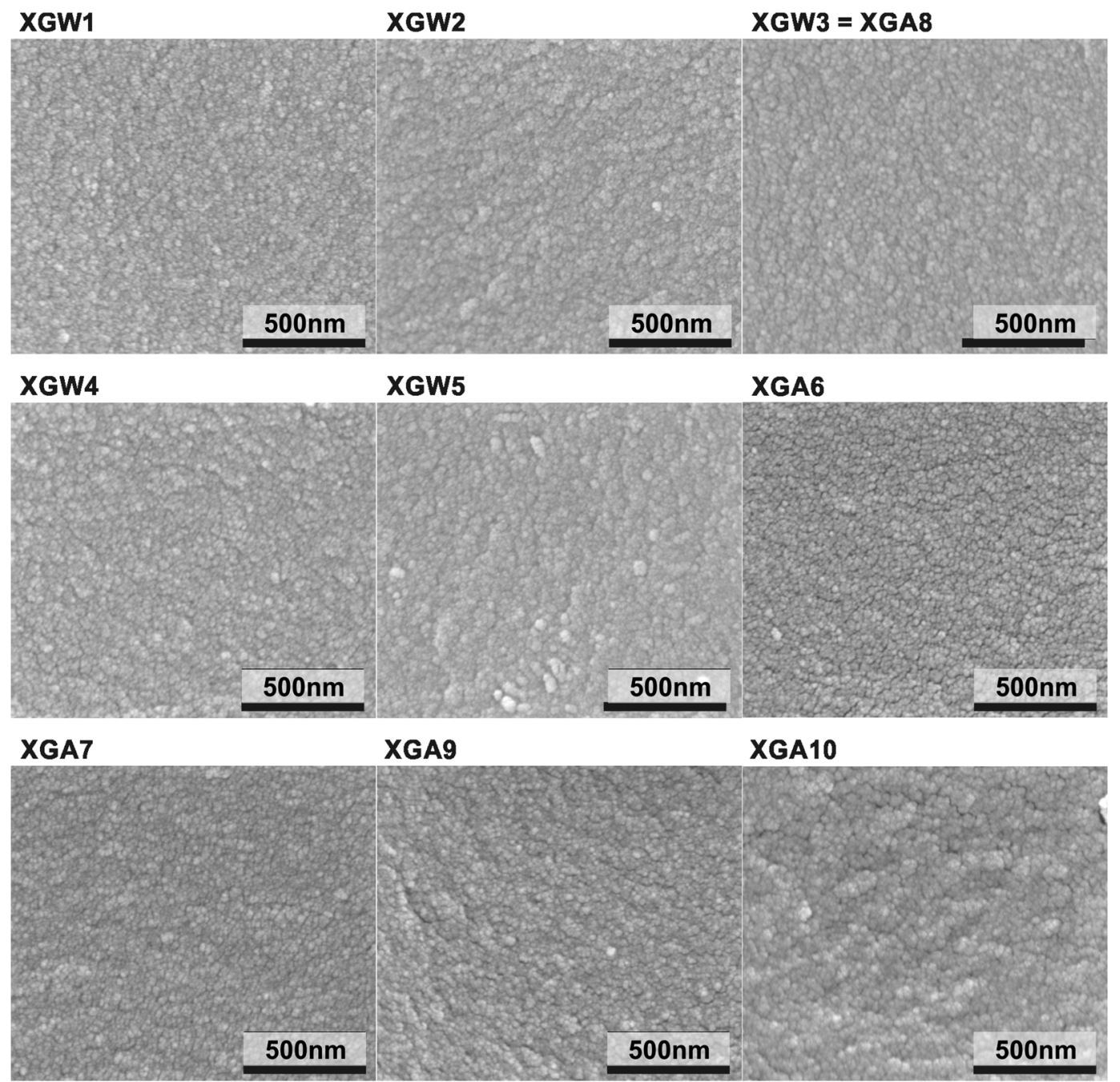

Fig. 1 Scanning electron microscopy images of the silica xerogels after drying

A low concentration of ammonia leads to the formation of smaller particles which are more susceptible to capillary pressure and can cause the network to deform and impinge one another. The result is a denser dry gel without any macropores [8].

A small water content leads also to the formation of smaller particles. With an increase in the water content, the particles of the xerogel become bigger which may be caused by an enhanced condensation rate $[34,36]$.

\subsection{Surface properties}

Figure 2a, $\mathrm{b}$ illustrate the $\mathrm{N}_{2}$ adsorption and desorption isotherms of the silica xerogels prepared with different ammonia concentration and different water content. The $\mathrm{N}_{2}$ isotherms of the silica xerogels show a high nitrogen uptake at low relative pressures $\left(\mathrm{P} / \mathrm{P}_{0}<0.1\right)$ and a plateau at high relative pressures which indicates the presence of microporosity. All samples (expect XGW1) show an additional uptake at relative pressures $\left(\mathrm{P} / \mathrm{P}_{0}>0.2\right)$ and a hysteresis, which reflects the presence of mesoporosity. The nitrogen sorption capacity increases with a higher ammonia concentration as well as with a higher water content (Fig. 2a, b). According to the BJH pore size distribution (Fig. 2c, d), the concentration of ammonia and the content of water increases the fraction of all pores indicating an increasing porosity.

The influence of the ammonia concentration and the water content on the BET surface area, micropore volume, mesopore volume, and the average pore size is shown in Table 3. It can be observed that the BET surface area increases with a higher concentration of ammonia or a higher amount of water. With an increase in the ammonia concentration, the mesopore volume significantly increases. An increase in water content during the condensation reaction increases the micropore volume as well as the 
Fig. 2 Low-temperature nitrogen adsorption (open symbols)/desorption (closed symbols) isotherms of silica xerogels with (a) different ammonia concentration and (b) different water content and its corresponding $\mathrm{BJH}$ pore size distributions (c, d)
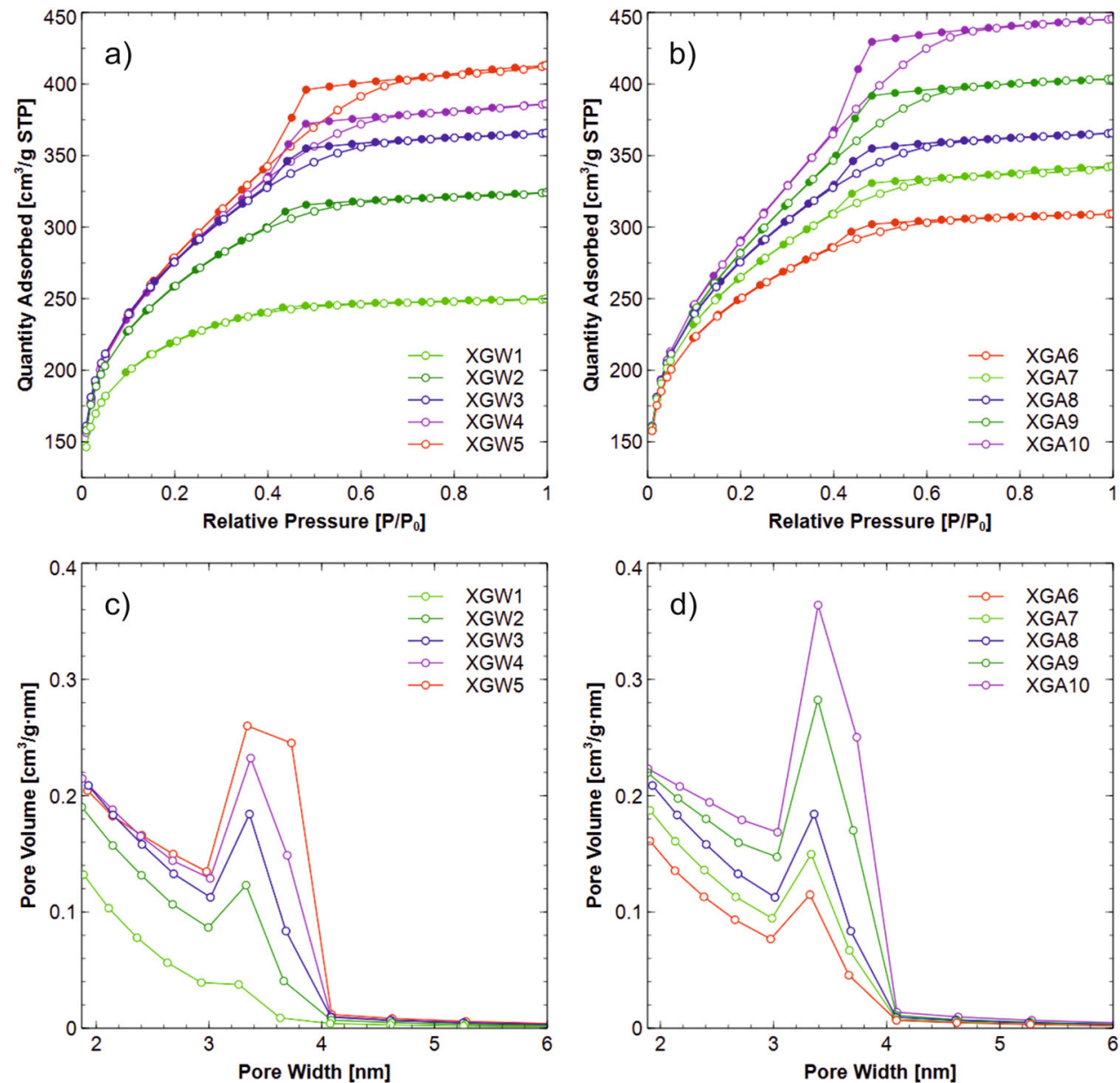

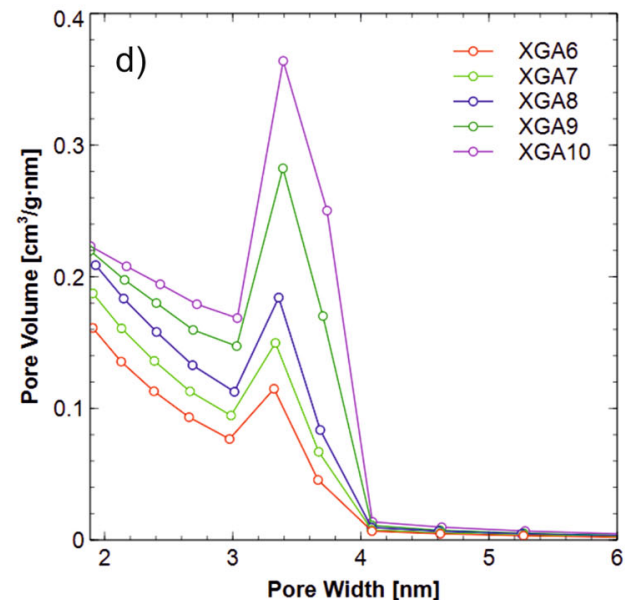

Table 3 BET surface area, micropore volume, mesopore volume, total pore volume and average pore size of the silica xerogels

\begin{tabular}{lcllll}
\hline Sample & $\begin{array}{l}\mathrm{S}_{\mathrm{BET}} \\
{\left[\mathrm{m}^{2} / \mathrm{g}\right]}\end{array}$ & $\begin{array}{l}\mathrm{V}_{\text {Micro }} \\
{\left[\mathrm{cm}^{3} / \mathrm{g}\right]}\end{array}$ & $\begin{array}{l}\mathrm{V}_{\text {Meso }} \\
{\left[\mathrm{cm}^{3} / \mathrm{g}\right]}\end{array}$ & $\begin{array}{l}\mathrm{V}_{\text {total }} \\
{\left[\mathrm{cm}^{3} / \mathrm{g}\right]}\end{array}$ & $\mathrm{D}_{\text {pore }}[\AA]$ \\
\hline XGW1 & 738 & 0.175 & 0.212 & 0.387 & 21.0 \\
XGW2 & 879 & 0.143 & 0.358 & 0.501 & 22.8 \\
XGW3 & 968 & 0.113 & 0.452 & 0.565 & 23.4 \\
XGW4 & 976 & 0.088 & 0.509 & 0.597 & 24.5 \\
XGW5 & 991 & 0.075 & 0.564 & 0.639 & 25.8 \\
XGA6 & 856 & 0.165 & 0.313 & 0.478 & 22.3 \\
XGA7 & 903 & 0.125 & 0.405 & 0.530 & 23.5 \\
XGA8 & 968 & 0.113 & 0.452 & 0.565 & 23.4 \\
XGA9 & 995 & 0.057 & 0.567 & 0.624 & 25.1 \\
XGA10 & 1039 & 0.038 & 0.651 & 0.689 & 26.5 \\
\hline
\end{tabular}

mesopore volume of the silica xerogels. These results are in agreement with the observations from Alcañiz et al. [2]. With an increase in the water:TEOS ratio, the micropore and mesopore volume increases whereas an increase in $\mathrm{pH}$ leads to a significant increase in the mesopore volume. The average calculated pore size increases with a higher concentration of ammonia and with a higher content of water. The pore volume calculated by the envelope and skeleton density (Table 2) is close to the total pore volume determined by the nitrogen sorption analysis. These results are in agreement with the observations from Fidalgo et al. [8] and Chan et al. [31]. An increase in the concentration of ammonia respectively $\mathrm{NaF}$ during gelation leads to an increase in average pore size and a higher total pore volume.

The full FTIR spectra $\left(4000 \mathrm{~cm}^{-1}-600 \mathrm{~cm}^{-1}\right)$ of all samples present in this study are shown in Fig. S1. The broad peak around $3400 \mathrm{~cm}^{-1}$ is ascribed to an $\mathrm{O}-\mathrm{H}$ stretching region where some freely vibrating $\mathrm{OH}$ groups and a majority of hydrogen-bonded $\mathrm{OH}$ groups are apparent [37]. Silica gels which contain physisorbed water show a small peak at $1630 \mathrm{~cm}^{-1}$ [38]. Since the silica xerogels in this study were degassed in a vacuum oven prior to the measurement, this feature is not visible. The FTIR spectra of the fingerprint region $\left(1400 \mathrm{~cm}^{-1}-600 \mathrm{~cm}^{-1}\right)$ of the silica xerogels synthesized with different ammonia concentrations are shown in Fig. 3a whereas the silica xerogels synthesized with different water contents are shown in Fig. 3b. The spectra look very similar. The adsorption band at $1090 \mathrm{~cm}^{-1}$ is related to asymmetric stretching modes of $\mathrm{Si}-\mathrm{O}-\mathrm{Si}$ bonds 

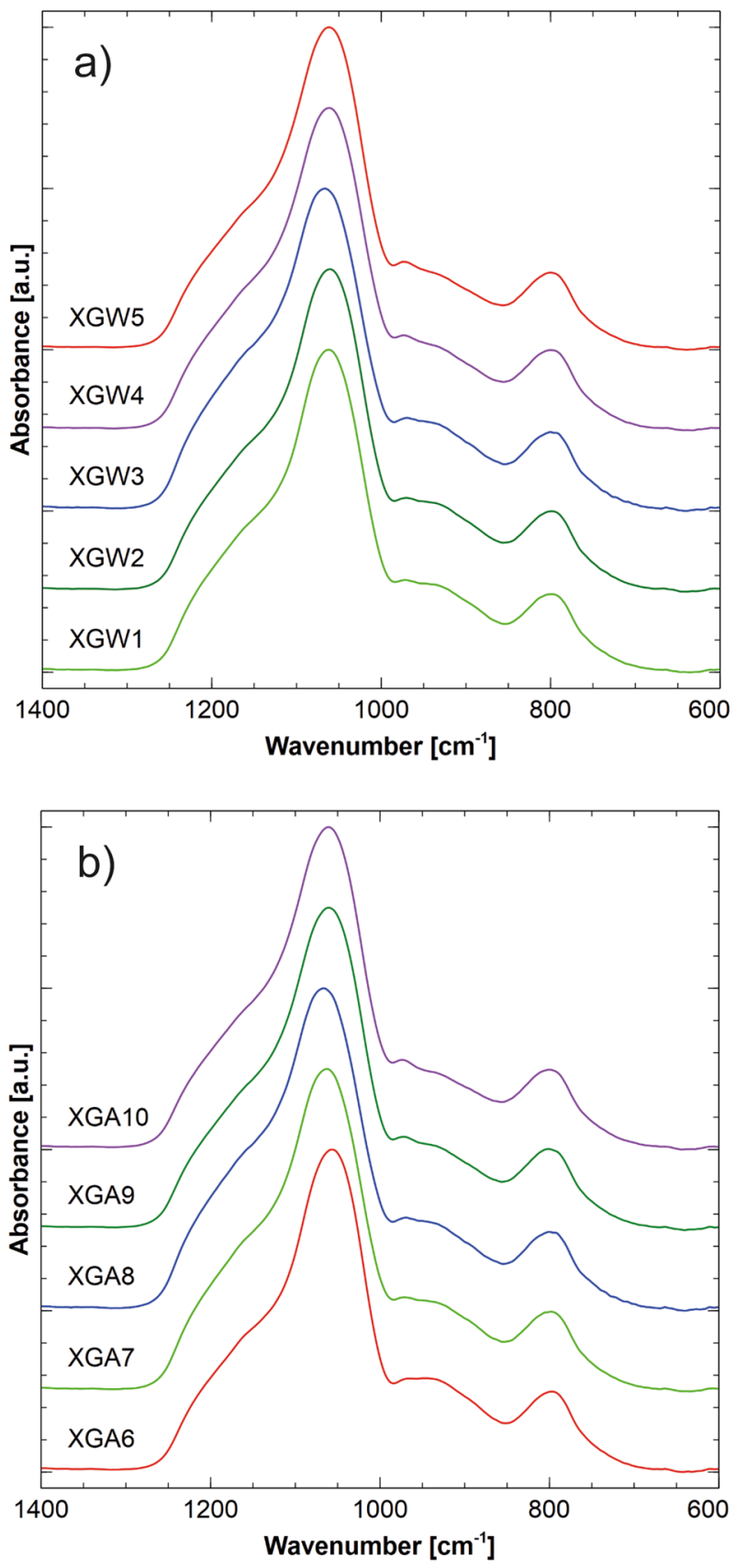

Fig. 3 FTIR spectra of silica xerogels with (a) different ammonia concentration and (b) different water content

[39]. The band at about $800 \mathrm{~cm}^{-1}$ is associated with the symmetric stretching modes of these bonds [40]. The band at $960 \mathrm{~cm}^{-1}$ reveals the presence of surface silanol groups on these samples [38, 40-42]. The intensity of the band at $960 \mathrm{~cm}^{-1}$ is higher for xerogels synthesized with a high concentration of ammonia and for xerogels which were synthesized with a high water content. Xerogels which were synthesized with a higher concentration of ammonia, respectively, with a higher content of water have a higher specific surface area and are therefore expected to have a greater number of nonbridging surface $-\mathrm{SiOH}$ groups [38]. The presence of these bands in the collected FTIR spectra confirms that sol-gel silica samples were successfully obtained in this study.

\subsection{Water sorption behavior}

The effect of the ammonia concentration on the water sorption behavior of the silica xerogels is shown in Fig. 4. A low concentration of ammonia leads to a higher water sorption at lower relative pressure and a small hysteresis, which might be due to the smaller pore size. However, the total water sorption capacity is relatively low, which is in good correlation with the BET surface area. A higher concentration of ammonia shifts the water sorption isotherm to higher relative pressure, which might be caused by the increasing pore width and the higher fraction of mesopores. Silica gels prepared with a higher content of water show an increase in water sorption capacity. These results are in agreement with findings from Ibrahem et al. [43] who found an increased water sorption capacity when the silica xerogels were synthesized with a higher $\mathrm{H}_{2} \mathrm{O} / \mathrm{TEOS}$ ratio. The increased water sorption capacity is consistent with the $\mathrm{N}_{2}$ sorption measurements which exhibited a larger BET surface area and a higher total pore volume for xerogels which were synthesized with a higher water content. Furthermore, it can be deduced that silica gels containing a higher mesopore volume exhibit a larger hysteresis of the water sorption isotherms.

The silica gel which was synthesized with the lowest concentration of ammonia (XGW1) has a higher water sorption capacity over the whole range of relative pressure compared to the reference silica gel (Fig. S2). The combination of a short processing time with the high water sorption capacity might be very interesting for industrial applications such as desiccants or AHPs.

\section{Conclusions}

Silica samples with promising applications as water sorbents were successfully obtained in this work. The total processing time, including hydrolysis, gelation, aging and ambient pressure drying is $10 \mathrm{~h}$. It was found that an increasing concentration of ammonia or a higher content of water leads to a significant reduction of the envelope density. Silica gels prepared from a higher concentration of ammonia exhibit a higher average pore size whereas the silica gels prepared from a higher content of water have an increased BET surface area. From the comparative analysis of nitrogen and water sorption isotherms, it was observed that water adsorption at low relative pressure mainly occurs in micropores. Silica gels containing a high fraction of 
Fig. 4 Water vapor adsorption (open symbols) and desorption (closed symbols) isotherms of silica gels prepared with different concentration of ammonia (XGW1-XGW5) and different amount of water (XGA6-XGA10)
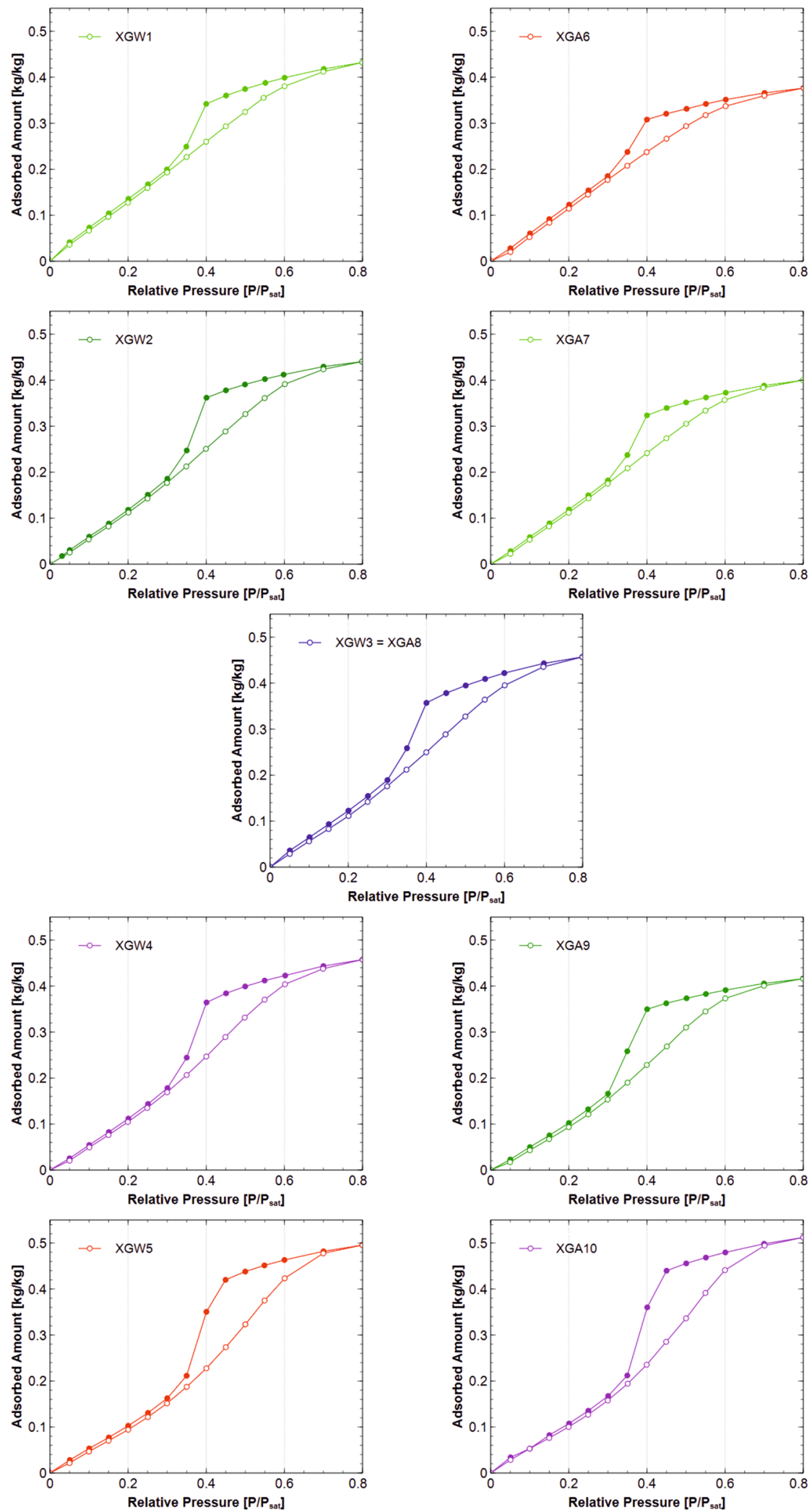

mesopores exhibit water adsorption at higher relative pressures compared with those containing a low fraction of mesopores. By a controlled addition of ammonia and water, silica xerogels can be tailored to have a higher water sorption capacity than the reference silica gel over the whole range of relative pressure. 
Acknowledgements The authors acknowledge the support of the Swiss National Science Foundation: National Research Programme (NRP70 under grant number 154008). We thank Beatrice Fischer for her assistance with the FTIR measurements. In addition, Christopher J. Ubert and Dr. Shanyu Zhao are acknowledged for editorial support. Open access funding provided by Empa - Swiss Federal Laboratories for Materials Science and Technology.

\section{Compliance with ethical standards}

Conflict of interest The authors declare that they have no conflict of interest.

Publisher's note Springer Nature remains neutral with regard to jurisdictional claims in published maps and institutional affiliations.

Open Access This article is licensed under a Creative Commons Attribution 4.0 International License, which permits use, sharing, adaptation, distribution and reproduction in any medium or format, as long as you give appropriate credit to the original author(s) and the source, provide a link to the Creative Commons license, and indicate if changes were made. The images or other third party material in this article are included in the article's Creative Commons license, unless indicated otherwise in a credit line to the material. If material is not included in the article's Creative Commons license and your intended use is not permitted by statutory regulation or exceeds the permitted use, you will need to obtain permission directly from the copyright holder. To view a copy of this license, visit http://creativecommons. org/licenses/by/4.0/.

\section{References}

1. Shimooka S, Yamazaki M, Takewaki T, et al. (2007) Improvement of water adsorptivity of activated carbon for adsorption heat pump by hydrophilic treatment. In: Proceedings of International Symposium on EcoTopia Science 2007, ISETS07, pp 174-177

2. Alcañiz-Monge J, Pérez-Cadenas M, Lozano-Castelló D (2010) Influence of pore size distribution on water adsorption on silica gels. J Porous Mater 17:409-416. https://doi.org/10.1007/s10934009-9317-0

3. Huber L, Ruch P, Hauert R et al. (2016) Monolithic nitrogen-doped carbon as a water sorbent for high-performance adsorption cooling. RSC Adv 6:25267-25278. https://doi.org/10.1039/C6RA00548A

4. Huber L, Ruch P, Hauert R et al. (2016) Water sorption behavior of physically and chemically activated monolithic nitrogen doped carbon for adsorption cooling. RSC Adv 6:80729-80738. https:// doi.org/10.1039/C6RA18660B

5. Xu R, Pang W, Yu J et al. (2010) Chemistry of zeolites and related porous materials: synthesis and structure. Chem Zeolites Relat Porous Mater Synth Struct. https://doi.org/10.1002/9780470822371

6. Henninger SK, Schmidt FP, Henning H-M (2010) Water adsorption characteristics of novel materials for heat transformation applications. Appl Therm Eng 30:1692-1702. https://doi.org/ 10.1016/j.applthermaleng.2010.03.028

7. Ohashi F, Maeda M, Inukai K et al. (1999) Study on intelligent humidity control materials: water vapor adsorption properties of mesostructured silica derived from amorphous fumed silica. J Mater Sci 4:1341-1346

8. Fidalgo A, Rosa ME, Ilharco LM (2003) Chemical control of highly porous silica xerogels: physical properties and morphology. Chem Mater 15:2186-2192

9. Fidalgo A, Ilharco LM (2004) Chemical tailoring of porous silica xerogels: local structure by vibrational spectroscopy. Chem - A Eur J 10:392-398. https://doi.org/10.1002/chem.200305079
10. Collins KE, De Camargo VR, Dimiras AB et al. (2005) Physisorbed water layer formation on fully hydroxylated mesoporous silicas. J Colloid Interface Sci 291:353-360. https://doi.org/10. 1016/j.jcis.2005.05.022

11. Wang D, Zhang J, Yang Q et al. (2014) Study of adsorption characteristics in silica gel - water adsorption refrigeration. Adsorpt J Int Adsorpt Soc 113:734-741. https://doi.org/10.1016/j. apenergy.2013.08.011

12. Li X, Li Z, Xia Q, Xi H (2007) Effects of pore sizes of porous silica gels on desorption activation energy of water vapour. Appl Therm Eng 27:869-876. https://doi.org/10.1016/j.applthermaleng. 2006.09.010

13. Hwang J, Kataoka S, Endo A, Daiguji H (2015) Adsorption and desorption of water in two-dimensional hexagonal mesoporous silica with different pore dimensions. J Phys Chem C 119:26171-26182. https://doi.org/10.1021/acs.jpcc.5b08564

14. Takei T, Yamazaki A, Watanabe T, Chikazawa M (1997) Water adsorption properties on porous silica glass surface modified by trimethylsilyl groups. J Colloid Interface Sci 188:409-414. https:// doi.org/10.1006/jcis.1997.4777

15. Feng A, McCoy BJ, Munir ZA, Cagliostro DE (1996) Water adsorption and desorption kinetics on silica insulation. J Colloid Interface Sci 180:276-284. https://doi.org/10.1006/jcis.1996.0300

16. Saliba S, Ruch P, Volksen W et al. (2016) Combined influence of pore size distribution and surface hydrophilicity on the water adsorption characteristics of micro- and mesoporous silica. Microporous Mesoporous Mater 226:221-228. https://doi.org/10. 1016/j.micromeso.2015.12.029

17. Hertl W, Hair ML (1969) Adsorption of water on silica. Nature 223:1150-1151. https://doi.org/10.1038/224488a0

18. Klier K, Shen JH, Zettlemoyer AC (1973) Water on silica and silicate surfaces. I. Partially hydrophobic silicas. J Phys Chem 77:1458-1465. https://doi.org/10.1021/j100630a026

19. Ugliengo P, Saunders V, Garrone E (1990) Silanol as a model for the free hydroxyl of amorphous silica - abinitio calculations of the interaction with water. J Phys Chem 94:2260-2267. https://doi. org/10.1021/j100369a013

20. Shen JH, Klier K (1980) Water on silica and silicate surfaces. V. Amorphous, porous, hydrophilic, phosphorus-doped silicas. J Colloid Interface Sci 75:56-67. https://doi.org/10.1016/00219797(80)90349-5

21. Hall PG, Pidduck A, Wright CJ (1981) Inelastic neutron scattering by water adsorbed on silica. J Colloid Interface Sci 79:339-349. https://doi.org/10.1016/0021-9797(81)90085-0

22. Horikawa T, Do DD, Nicholson D (2011) Capillary condensation of adsorbates in porous materials. Adv Colloid Interface Sci 169:40-58. https://doi.org/10.1016/j.cis.2011.08.003

23. Miyahara M, Kanda H, Yoshioka T, Okazaki M (2000) Modeling capillary condensation in cylindrical nanopores: a molecular dynamics study. Langmuir 16:4293-4299. https://doi.org/10.1021/la 991227e

24. Ng KC, Chua HT, Chung CY et al. (2001) Experimental investigation of the silica gel-water adsorption isotherm characteristics. Appl Therm Eng 21:1631-1642. https://doi.org/10.1016/S13594311(01)00039-4

25. Satoh S, Matsuyama I, Susa K (1995) Diffusion of gases in porous silica gel. J Non Cryst Solids 190:206-211. https://doi.org/10. 1016/0022-3093(95)00275-8

26. Aristov YI, Tokarev MM, Freni A et al. (2006) Kinetics of water adsorption on silica Fuji Davison RD. Microporous Mesoporous Mater 96:65-71. https://doi.org/10.1016/j.micromeso.2006.06.008

27. Rodrigues Mota TL, Marques de Oliveira AP, Nunes EHM, Houmard M (2017) Simple process for preparing mesoporous solgel silica adsorbents with high water adsorption capacities. Microporous Mesoporous Mater 253:177-182. https://doi.org/10. 1016/j.micromeso.2017.07.010 
28. Brewer CE, Chuang VJ, Masiello CA et al. (2014) New approaches to measuring biochar density and porosity. Biomass and Bioenergy 66:176-185. https://doi.org/10.1016/j.biombioe.2014.03.059

29. Brunauer S, Emmett PH, Teller E (1938) Gases in multimolecular Layers. J Am Chem Soc 60:309-319

30. Lippens BC, de Boer JH(1965) Studies on pore systems in catalysts: V. The t method. J Catal 4:319-323

31. Chan J-B, Hua D-W, Winter R, Jonas J (1989) The pore morphology of fluoride catalyzed xerogels. J Mater Res 4:693-697. https://doi.org/10.1557/JMR.1989.0693

32. Rodríguez-Reinoso F, Molina-Sabio M, Gonzalez MT (1995) The use of steam and $\mathrm{CO} 2$ as activating agents in the preparation of activated carbons. Carbon N Y 33:15-23

33. Barrett EP, Joyner LG, Halenda PP (1951) The determination of pore volume and area distributions in porous substances. I. Computations from nitrogen isotherms. J Am Chem Soc 73:373-380. https://doi.org/10.1021/ja01145a126

34. Milea CA, Bogatu C (2011) The influence of parameters in silica sol-gel process. Bull Transilv Univ Brasov Eng Sci 4:59-66

35. Hæreid S, Nilsen NEM-A (1996) Properties of silica aerogel aged in TEOS. J Non Cryst Solids 204:228-234

36. Scherdel C, Reichenauer G (2015) Highly porous silica xerogels without surface modification. J Supercrit Fluids 106:160-166. https://doi.org/10.1016/j.supflu.2015.08.016
37. Almeida RM, Pantano CG (1990) Structural investigation of silica gel films by infrared spectroscopy. J Appl Phys 68:4225-4232. https://doi.org/10.1063/1.346213

38. Wood DL, Rabinovich EM (1989) Study of alkoxide silica gels by infrared spectroscopy. Appl Spectrosc 43:263-267. https://doi. org/10.1366/0003702894203282

39. Román J, Padilla S, Vallet-Regí M (2003) Sol-gel glasses as precursors of bioactive glass ceramics. Chem Mater 15:798-806. https://doi.org/10.1021/cm021325c

40. Fidalgo A, Ilharco LM (2001) The defect structure of sol-gelderived silica/polytetrahydrofuran hybrid films by FTIR. J Non Cryst Solids 283:144-154. https://doi.org/10.1016/S0022-3093 (01)00418-5

41. Andrade-Espinosa G, Escobar-Barrios V, Rangel-Mendez R (2010) Synthesis and characterization of silica xerogels obtained via fast sol-gel process. Colloid Polym Sci 288:1697-1704. https://doi.org/10.1007/s00396-010-2311-x

42. Gaskell PH, Johnson DW (1976) The optical constants of quartz, vitreous silica and neutron-irradiated vitreous silica (I). J Non Cryst Solids 20:153-169. https://doi.org/10.1016/0022-3093(76) 90130-7

43. Seenaa Ibrahem HI (2014) Synthesis and study the effect of H2O/ TEOS ratio of the silica xerogel by sol- gel method. Int Arch Appl Sci Technol 5:1-5 